\title{
Psychometric Properties of a Persian-Language Version of the Obsessive-Compulsive Inventory-Revised: OCI-R-Persian
}

\author{
Habibollah Ghassemzadeh $^{1 *}$, Giti Shams ${ }^{1}$, Jamal Abedi ${ }^{2}$, Narges Karamghadiri ${ }^{1}$, \\ Narges Ebrahimkhani ${ }^{1}$, Mansoureh Rajabloo ${ }^{3}$ \\ ${ }^{1}$ Roozbeh Hospital, Department of Psychiatry Tehran University of Medical Sciences, Tehran, Iran; \\ ${ }^{2}$ School of Education, University of California, Davis, USA; \\ ${ }^{3}$ Private practice, Tehran, Iran. \\ *Email: hghassemzadeh@tums.ac.ir \\ Received October 17 $7^{\text {th }}, 2010$; revised March $4^{\text {th }}, 2011$; accepted April $5^{\text {th }}, 2011$.
}

\begin{abstract}
The psychometric properties of the Persian-language version of Obsessive-Compulsive Inventory-Revised $(\mathrm{OCI}-\mathrm{R})$ were studied in a sample of Iranian college students $(\mathrm{N}=450)$. The total and each of the subscales of OCI-R-Persian demonstrated very high internal consistency as well as high test-retest reliability. Convergent and divergent validity of the OCI-R-Persian total scale and subscales were satisfactory. In general, the OCI-R-Persian appears to be a reliable and valid measure of obsessive-compulsive symptoms in this non-clinical sample of Iranian college students.
\end{abstract}

Keywords: Confirmatory Factor Structure, OCD, OCI-R-Persian, Psychometric Properties

\section{Introduction}

The utility of using psychological measures of obsessivecompulsive disorder (OCD) in both research and clinical settings is widely recognized (Goodman and Price, 1998; St. Clare, 2003). Many of these measures are based on self-report of symptoms. In a review of different self report measures, Foa, Kozak and Salkovskis (1998) addressed some of the problems and limitations inherent in such instruments as Yale-Brown Obsessive-Compulsive Scale (YBOCS; Goodman et al., 1989), Layton Obsessional Inventory (LOI; Taylor, 1995), the Compulsive Activity Checklist (CAC; Cottraux et al., 1988), and the Maudsley Obsessive-Compulsive Inventory (MOCI; Hodgson \& Rachman, 1977). Based on their critical review, Foa and her colleagues (1998) introduced a new obsessive-compulsive disorder scale called the Obsessive-Compulsive Inventory (OCI). This scale consists of 42 items grouped in seven subscales (checking, washing, obsessing, mental neutralizing, ordering, hoarding and harming, and doubting). The subscales are further divided up into frequency and distress dimensions.

The OCI showed "excellent" internal consistency and "satisfactory" test-retest reliability for both symptom frequency and associated distress in individuals with OCD and in non-patient controls. The coefficient alphas for OCI total score in OCD group were .93 for frequency and .92 for distress. The same coefficients were .94 and .95 in control group. The test-retest reliabilities for OCI total score in OCD group were 84 for frequency and .87 for distress. The same coefficients were .90 and .89 in control group (Foa et al., 1998). These researchers further introduced a revised shorter version of the OCI, the OCI-R. Several considerations led to the revision: 1) there was a high correlation between the distress and frequency total score (above .90) in the original OCI, indicating redundancy in the two scales; 2) the time needed for the completion of OCI made it somewhat difficult to use in the clinical and/or hospital settings; and 3) the number of items varied across scales in the original OCI. The OCI-R consists of 18 items, yielding six subscales: washing, checking, ordering, obsessing, hoarding, and neutralizing. It is significantly shorter than OCI, and eliminates the frequency scale. Each subscale has three items which are summed up, facilitating comparisons across scales. The psychometric properties of the OCI-R were assessed by Foa and her colleagues (2002). The subsequent studies have provided further support for the psychometric properties of OCI-R in both clinical and non-clinical samples (e.g., Hajcak et al., 2004; Abramowitz \& Deacon, 2006; Smari et al., 2007).

\section{OCD Measures in Iran}

A number of instruments for assessment of OCD symptoms have been previously translated into Persian (Farsi) and used in Persian speaking patient and non-patient populations. In an earlier study (Ghassemzadeh et al., 2002) we examined the symptoms in a sample of Iranian OCD patients using Persian translations of the Maudsley Obsessional-Compulsive Inventory (MOCI) and Compulsive Activity Checklist (CAC). In our study, the mean scores on MOCI $(M=15.73, S D=5.63)$ and CAC $(M=14.90, S D=9.77)$, were close to those reported in other settings. However, we encountered numerous problems in using MOCI in this setting. First, some of the items were not easily understood by the patients, especially the double-negative sentences (e.g. "I don't worry unduly about contamination if I touch an animal."). Second, the true-false response format of MOCI made it difficult for many indecisive patients to respond. Third, as Foa et al. (1998) suggested the items of the MOCI encompass only two of the behavioral compulsions (i.e. 
checking and washing) and do not measure specific obsessions other than contamination. These difficulties with MOCI motivated us to translate and examine the feasibility of the OCI-R in the Iranian culture. In our preliminary work, we found OCI-R practical and easily understood by most respondents. Having fewer items than MOCI, using a 5-point Likert scale response format, and equal number items for every subscale are other important aspects of OCI-R. In this report, we present the results of a study of the psychometric properties and construct validity of the Persian translation of the OCI-R in a sample of 450 Iranian male and female college students.

\section{Method}

\section{Participants}

The sample consisted of 450 (164 males and 286 females) student volunteers recruited from Tehran University of Medical Sciences and Allameh Tabatabai University, both in Tehran, Iran. The average age of the total sample was $22.12(S D=3.95)$ for males and $22.83(S D=3.53)$ for females.

\section{Measures}

\section{Obsessive-Compulsive Inventory-Revised (OCI-R)}

The OCI-R is an 18-item instrument developed based on the earlier 42-item version (Foa, Kozak, \& Salkovskis et al., 1998; Foa, Huppert, \& Leiberg et al., 2002). The OCI-R comprises six scales: (1) Washing, (2) Checking/doubting, (3) Obsessing, (4) Mental neutralizing, (5) Ordering, and (6) Hoarding. Each item is rated on a five-point (0 - 4) Likert scale for distress or bother during the past month. The total score ranges from 0 to 72 .

The original English version of OCI-R was obtained from Dr. Edna Foa at the University of Pennsylvania and translated to Persian by the first author (H. G.). The Persian draft was then back-translated by a professional bilingual translator. The back translated version was then sent to one of the colleagues of Dr. Foa who reviewed the English text and provided suggestions to improve the accuracy of the Persian translation. After incorporating those points in the translation and going through to the process of second back translation, the final version of OCI-R Persian was ready to use in our sample.

\section{Mausley Obsessional-Compulsive Inventory (MOCI)-Persian version}

The MOCI is a 30 -item true-false instrument for assessment of OCD symptoms. Maximum scores for the five scales (total, checking, washing, slowness-repetition, and doubt-conscientious) are $30,9,11,7$, and 7 , respectively. This inventory has been used with adequate validity and reliability (Rachman \& Hodgson, 1980). The Persian language version of the test has been used in a number of studies in Iran (Ghassemzadeh, 2002, 2005a, 2005b).

\section{Compulsive Activity Checklist (CAC)}

The original format of this inventory had 62 items concerning specific daily activities rated on a 4-point scale of severity. We used a French version of CAC, which is an abbreviated form of the instrument and contains 19 items centered mainly on checking behavior and rituals (Cottraux et al., 1988). Each item is rated on a four-point $(0-3)$ scale, therefore the scores range from 0 to 57 . Different versions of CAC have demonstrated good internal consistency (St. Clare, 2003). This test has been translated into Persian and used in our previous studies in Iran (Ghassemzadeh et al., 2002, 2005b).

\section{Beck Depression Inventory-II- Persian Version}

BDI-II is the second edition of the widely used 21-item inventory, the BDI. Originally developed in 1960s (Beck et al., 1961), BDI-II was revised to approximate the DSM-IV criteria for major depression. Scores on each item range from 0 to 3, yielding a possible maximum total score of 63. The Persian version of this test (BDI-II- Persian) is widely used in various settings in Iran. Its psychometric properties have been examined elsewhere (Ghassemzadeh, et al., 2005c).

\section{Procedure}

All the tests were administered to the students in a group setting during a class session after a brief description of the purpose of the study. The students were told that the participation is voluntary and no payment or course credit was offered to the participants. The OCI-R was administered first, followed by MOCI, CAC and BDI-II. All the assessments were repeated in the same sample after 2 weeks. Due to some logistical issues, MOCI, CAC and BDI-II were administered only to 228 subjects of the total sample.

\section{Results}

\section{Descriptive Statistics of the OCI-R Scale and Subscales}

The means and standard deviations on the OCI-R (total and subscales) for males and females (separately and total) are presented in Table 1.

As the data in Table 1 show, female students generally scored higher than males on most subscales. However, the difference reached a statistically significant level only on the "obsessing" subscale $(t=2.27, \mathrm{~d} f=448, P<.05)$.

Table 2 shows the mean scores obtained in the present study as compared with Foa's group (2002). Our mean score for the whole group is very close to their mean score (18.46 vs. 18.82). This is true for some subscales but with some considerations. Since Foa's group was regarded as non-anxious control and our group as normal college students, it would be difficult to compare the results of these two studies.

\section{Correlations among OCI-R Subscales}

Correlations between each of the subscales and the total scale of the OCI-R as well as the inter-correlations among the subscales are shown in Table 3. Correlations between each of the subscales and the total scale ranged from .63 (ordering) to .76 (obsessing). Inter-correlations among the subscales ranged from .21 (neutralizing \& ordering) to .51 (hoarding \& obsessing). All the correlations were significant at .01 level.

\section{Test-Retest Reliability and Internal Consistency}

The two-week test-retest reliability was calculated using Pearson's $r$ correlation coefficients. Cronbach's alpha was calculated and used to assess the internal consistency of the OCI-R 
Table 1.

Scores on the OCI-R scale among 450 female and male Iranian college students.

\begin{tabular}{|c|c|c|c|c|c|c|c|}
\hline \multirow{2}{*}{ OCI-R } & \multicolumn{2}{|c|}{$\begin{array}{c}\text { Total } \\
(\mathrm{N}=450)\end{array}$} & \multicolumn{2}{|c|}{$\begin{array}{l}\text { Females } \\
(\mathrm{N}=286)\end{array}$} & \multicolumn{2}{|c|}{$\begin{array}{c}\text { Males } \\
(\mathrm{N}=164)\end{array}$} & \multirow[t]{2}{*}{$\begin{array}{c}t \\
(\mathrm{~d} f=448)\end{array}$} \\
\hline & $M$ & S.D. & $M$ & S.D. & $M$ & S.D. & \\
\hline Checking & 2.38 & 2.26 & 2.42 & 2.35 & 2.32 & 2.09 & .45 \\
\hline Hoarding & 3.49 & 2.49 & 3.59 & 2.59 & 3.30 & 2.30 & 1.18 \\
\hline Neutralizing & 1.09 & 1.74 & 1.11 & 1.72 & 1.07 & 1.79 & .24 \\
\hline Ordering & 5.16 & 2.87 & 5.13 & 2.96 & 5.22 & 2.70 & -.33 \\
\hline Washing & 2.19 & 2.21 & 2.24 & 2.28 & 2.11 & 2.09 & .59 \\
\hline Obsessing & 4.15 & 2.90 & 4.38 & 3.01 & 3.74 & 2.65 & $2.27^{*}$ \\
\hline Total & 18.46 & 9.92 & 18.86 & 10.44 & 17.76 & 8.93 & 1.13 \\
\hline
\end{tabular}

$*_{p}<.05$.

Table 2.

The mean and SD scores of OCI-R (total and subscales) in the present study and Foa's mean and SD scores for non-anxious controls (NACs).

\begin{tabular}{cccccc}
\hline & \multicolumn{3}{c}{$\begin{array}{c}\text { Foa's group } \\
(\mathrm{N}=477)\end{array}$} & \multicolumn{2}{c}{$\begin{array}{c}\text { Present study } \\
(\mathrm{N}=450)\end{array}$} \\
\cline { 2 - 5 } OCI-R & $M$ & S.D. & $M$ & 2.38 \\
\hline Checking & 2.91 & 2.56 & 3.49 & 2.38 & 2.47 \\
Hoarding & 4.41 & 2.67 & 1.09 & 5.16 & 2.74 \\
Neutralizing & 1.82 & 2.20 & 2.19 & 2.87 \\
Ordering & 4.40 & 3.03 & 4.15 & 2.21 \\
Washing & 2.41 & 2.50 & 18.46 & 2.90 \\
Obsessing & 2.86 & 2.72 & 11.10 & & 9.92 \\
Total & 18.82 & & & \\
\hline
\end{tabular}

Table 3.

Correlations between OCI-R subscales and total scale and inter-correlations among the subscales.

\begin{tabular}{|c|c|c|c|c|c|c|c|}
\hline OCI-R & Checking & Hoarding & Neutralizing & Ordering & Washing & Obsessing & Total \\
\hline Checking & 1 & .47 & .26 & .31 & .48 & .49 & .73 \\
\hline Hoarding & & 1 & .32 & .29 & .41 & .51 & .73 \\
\hline Neutralizing & & & 1 & .21 & .22 & .31 & .51 \\
\hline Ordering & & & & 1 & .36 & .29 & .63 \\
\hline Washing & & & & & 1 & .42 & .70 \\
\hline Obsessing & & & & & & 1 & .76 \\
\hline Total & & & & & & & 1 \\
\hline
\end{tabular}

All p-value $<.01$.

total scale, as well as the subscales (Table 4). The OCI-R total scale as well as its subscales demonstrated very high test-retest correlations and internal consistency.

\section{Convergent and Divergent Validity of the OCI-R Total Scale and Subscales}

Convergent validity of OCI-R total scale was determined by correlating the OCI-R with two other measures of obsessivecompulsive symptoms, the MOCI and CAC, which have been used in the previous studies of OCD in this setting (Ghassemzadeh et al., 2002, 2005a). The correlations between the OCI-R total and subscale scores with MOCI total, washing and checking subscales are presented in Table 5.

The MOCI and the CAC total scores correlated .571 and .475 , 
respectively with OCI-R, indicating moderate convergent validity.

Correlations of OCI-R checking and washing subscales with corresponding subscales of MOCI were .558 and .338 , respectively. But the highest correlation (.575) was related to MOCI total score with OCI-R checking subscale. Correlations among different subscales of OCI-R and MOCI washing and checking subscales varied from .130 to .575 .

The divergent validity of OCI-R was assessed by correlating the OCI-R total and subscale scores with BDI-II (Table 6). The correlation between OCI-R total and BDI-II as a distinct measure from OCD was .398 , indicating a lower correlation than the convergent validity.

\section{Factor Structure of OCI-R-Persian}

A confirmatory factor analytic model was used to examine the conceptual structure underlying the OCI-R, the Persian version. These analyses were conducted using EQS software (Bentler, 1992). Two SEM models were created. In the first model all the subscales include 3 items- the same as Foa's (Foa et al., 2002) findings except "neutralizing" which includes 2 items (item 16 was excluded) and washing which includes 4 items (item 16 was added to this subscale). Item 16 in the original Foa's scale was part of the "neutralizing" subscale.

Table 4.

Test-retest reliability and internal consistency coefficients for OCI-R total scale and subscales.

\begin{tabular}{ccc}
\hline OCI-R & Test-retest & Cronbach's alpha \\
\hline Checking & .70 & .82 \\
Hoarding & .73 & .84 \\
Neutralizing & .62 & .77 \\
Ordering & .69 & .82 \\
Washing & .66 & .80 \\
Obsessing & .76 & .86 \\
Total & .75 & .85 \\
\hline
\end{tabular}

Table 5.

Convergent validity for the OCI-R-Persian subscale and total scores.

\begin{tabular}{ccccc}
\hline \multirow{2}{*}{$\begin{array}{c}\text { OCI-R } \\
\text { N }=450)\end{array}$} & \multicolumn{2}{c}{$(\mathrm{N}=228)$} & \multicolumn{2}{c}{$\begin{array}{c}\text { CAC } \\
(\mathrm{N}=228)\end{array}$} \\
\cline { 2 - 5 } & Total & Washing & Checking & Total \\
\hline Checking & .575 & .314 & .558 & .359 \\
Hoarding & .378 & .193 & .291 & .365 \\
Neutralizing & .191 & .130 & .185 & .275 \\
Ordering & .223 & .184 & .137 & .111 \\
Washing & .394 & .338 & .333 & .412 \\
Obsessing & .524 & .300 & .527 & .397 \\
Total & .571 & .365 & .506 & .475 \\
\hline
\end{tabular}

Table 6.

Divergent Validity for OCI-R-Persian.

\begin{tabular}{cc}
\hline OCI-R & BDI-II \\
$(\mathrm{N}=450)$ & .381 \\
Checking & .244 \\
Hoarding & .146 \\
Neutralizing & .137 \\
Ordering & .203 \\
Washing & .457 \\
Obsessing & .398 \\
Total
\end{tabular}

Alternatively, the second model included item 16 in the "neutralizing" subscale as was originally suggested by Foa. Table 7 presents the results of SEM analyses for Model 1 and Table 8 reports the results for Model 2. For both models, we have assessed all the fit indices reported in the SEM literature including Normed Fit Index (NFI), Comparative Fit Index (CFI), Joreskog-Sorbom's GFI and standardized Root Mean-Square Residuals (SRMR) (Bentler \& Bonett, 1980; Bollen, 1989). As it can be seen from both Tables 7 and 8, these fit indices are all at the satisfactory level and suggest a good fit of the data to the models. For model 1 the NFI is .876 and the GFI for this model is .928 both suggesting a good fit. Similarly, for model 2, the NFI is .876 and the GFI is .929 almost identical with the fit indices of Model 1. Other fit indices reported for the two models are also almost identical suggesting that both models fit equally to the data: $\mathrm{CFI}=.919$ for both models; SRMR $=.049$ (model 1) and .054 (model 2); RMSEA $=.060$ for both models. It is usually believed that NFI, CFI, and GFI values of .90 or greater and SRMR and RMSEA values of .10 or less are markers of an acceptable fit (Hu \& Bentler, 1999). These values in our study are in an acceptable range.

However, examination of the factor structure of the two models reveals that item 16 does not fit well under any of the two models. In fact, this item has the lowest loading with its latent variable ("ordering" or "neutralizing") than any other items with their respective latent variables in both models. This item does not share a common variance with many other items included in the inventory. Therefore, it does not make much difference to include it in either "ordering" or "neutralizing" subscales. There might be some issue with this item, which will be considered in discussion section.

Considering the fact that our results, in general, support the original subscales developed by Foa's group (2002), we analyzed our data based on a six-factor model.

\section{Discussion}

Overall, the OCI-R appears to have excellent psychometric properties in this relatively large sample of Iranian college students. This finding is consistent with those from a number of earlier studies examining the psychometric properties of OCI-R in non-clinical samples from the US (Foa et al., 2002; Hajcak et al., 2004), Spain (Fullana et al., 2005), and Iceland (Smari et al., 
2007). Our work extends this line of investigation by examining the psychometric properties of OCI-R in a non-Western setting.

The OCI-R-Persian has excellent internal consistency (alpha ranges from .77 to .86 ) and test-retest reliability (correlations ranges from .62 to .76). The correlations between subscales and total scale vary between .51 and .76 , indicating a large correlation. But inter-correlations between the subscales were only moderate. The lowest inter-correlations were related to neutralizing. The test-retest reliability for total scale was .75. Although the coefficients were smaller than Foa's group (2002),

Table 7.

Model 1, with 6 factors, item 16 loading on "ordering".

\begin{tabular}{lcc}
\hline \multirow{2}{*}{ Factors (Subscales) } & Questions & Factor Structures \\
\hline \multirow{3}{*}{ 1. Checking } & Q14 & .663 \\
& Q 2 & .706 \\
2. Hoarding & Q 8 & .594 \\
& Q 7 & .686 \\
3. Neutralizing & Q 1 & .607 \\
& Q13 & .685 \\
4. Ordering & Q 4 & .742 \\
& Q10 & .832 \\
5. Washing & Q16 & .276 \\
& Q 3 & .797 \\
& Q15 & .802 \\
6.Obsessing & Q 9 & .631 \\
& Q17 & .604 \\
& Q 5 & .474 \\
& Q11 & .626 \\
& Q 6 & .653 \\
& Q12 & .782 \\
& Q18 & .775 \\
\hline
\end{tabular}

Table 8 .

Model 2, with 6 factors, item 16 loading on "neutralizing".

\begin{tabular}{lcc}
\hline \multicolumn{1}{c}{ Factors (Subscales) } & Questions & Factor Structures \\
\hline \multirow{3}{*}{ 1. Checking } & Q14 & .661 \\
& Q 2 & .707 \\
2. Hoarding & Q 8 & .594 \\
& Q 7 & .686 \\
3. Neutralizing & Q 1 & .609 \\
& Q13 & .684 \\
4. Ordering & Q 4 & .269 \\
& Q10 & .793 \\
5 & Q16 & .774 \\
5. Washing & Q 3 & .799 \\
& Q15 & .800 \\
& Q 9 & .632 \\
6. Obsessing & Q17 & .596 \\
& Q 5 & .472 \\
& Q11 & .631 \\
& Q 6 & .655 \\
& Q12 & .782 \\
& Q18 & .774 \\
\hline
\end{tabular}

all of them indicated excellent test-retest reliability. Our testretest reliabilities were in general somewhat higher than the scores reported in Hajcak's et al. (2004). For example, their obtained value for total scale was .70 , which was lower than our value (.75). But their reported values for washing, checking and obsessing were somewhat higher than our values. Fullana's et al. (2005) obtained a test-retest reliability of .67 which is lower than our value. Their obtained scores for the subscales were also smaller than our scores.

Similar to other studies (e.g. Fullana, et al., 2005), we found that OCI-R correlated strongly with other measures of OCD symptoms (such as MOCI and CAC). Satisfactory convergent validity is indicated by positive correlation of OCI-R-Persian total score with the total scores of MOCI and CAC (.571 and .475 , respectively). Furthermore, the checking subscale of the OCI-R-Persian and MOCI was also positively correlated ( $\mathrm{r}$ $=.558)$, but the correlation between two washing subscales of OCI-R-Persian and MOCI was not very high $(\mathrm{r}=.338)$. This may be partly related to the emphasis on obsessionality in comparison to compulsivity in OCI-R as compared to MOCI. Another reason may be related to the differences between the formats of two tests. The OCI-R is scored on a Lickert-scale whereas the MOCI is scored with a true-false format.

Apart from the problem of item 16, neutralizing subscale itself is somewhat questionable in the original version of OCI-R. The Cronbach's alpha for this subscale was .34 (the lowest), while the coefficients for the total scale and other subscales were all high, ranging from .65 to .89 in Foa et al. study (2002). Although in our study Cronbach's alpha for neutralizing was good $(\mathrm{r}=.77)$, it was lowest as compared to other subscales. We found the same trend in test-retest reliability (Table 4). The correlation between this subscale with the total scale in our study was .51 , while the correlations for other subscales with the total scale ranged from .63 to .76. As Abramowitz and Deacon (2006) have indicated the findings suggest that the neutralizing subscale is misnamed and possesses weaker construct validity than other OCI-R subscales. It may measure completely different construct than other items or the wording (linguistic structure) of the item is complex creating some difficulties in comprehending this item, or there might be some unique cultural aspect to this item that may not provide the same meaning across different subgroups. Therefore, this item can be considered as "attention" item that needs a careful review and inspection.

We found that female students had somewhat higher OCI-RPersian total and subscales scores than males but this did not reach statistical significance, except in the case of obsessing $(t$ $=2.27, \mathrm{~d} f=448, P<.05)$. No systematic evaluation of sex differences were provided in the past OCI-R research (Fullana et al., 2005). In our earlier studies with OCD patients in Iran, we found significant differences between females and males ( $\mathrm{F}$ $>\mathrm{M}$ ) in the total mean scores as well as in washing subscale of MOCI. The same trend was found in CAC, but not in Y-BOCS total or subscale scores (Ghassemzadeh et al., 2002, 2005b).

In conclusion, we found the OCI-R-Persian to be a brief and time-efficient measure of obsessions and compulsions in the general population in this setting. Participants had no difficulty in understanding the questions. Thus, it is an ideal measure for use for screening and epidemiological research in large general population samples. 
Future work needs to examine the psychometric properties of OCI-R-Persian in clinical samples and its utility as a measure of change of OCD symptomatology in clinical setting (c.f. Abramowitz \& Deacon, 2006). Future work also needs to assess the potential utility of OCI-R in distinguishing OCD from other types of anxiety disorders and depression in this setting.

\section{References}

Abramowitz, J. S., \& Deacon, B. J. (2006). Psychometric properties and construct validity of Obsessive- Compulsive Inventory-Revised: Replication and extension with a clinical sample. Anxiety Disorders, 20, 1016-1035. doi:10.1016/j.janxdis.2006.03.001

Beck, A. T., Ward, C. H.; Mendelsohn, M., Mock, J., \& Erlbaugh, J. (1961). An inventory for measuring depression. Archives of General Psychiatry, 4, 561-571.

Bentler, P. M. (1992). EQS structural equations program manual. Los Angeles: BMDP Statistical Software.

Bentler, P. M., \& Bonett, D. G. (1980). Significance tests and goodness of fit in the analysis of covariance structures. Psychological Bulletin, 88, 588-606. doi:10.1037/0033-2909.88.3.588

Bollen, K. A. (1989). Structural equations with latent variables. New York: John Wiley \&Sons, Inc.

Cottraux, J., Bouvard, M., Defayolle, M, \& Messy, P. (1988). Validity and factorial structure the Compusive Activity Checklist. Behavior Therapy, 19, 45-53. doi:10.1016/S0005-7894(88)80054-6

Foa, E. B., Huppert, J. D., Leiberg, S., Hajcak, G., Langner, R., Kichic, R., \& Salkovskis, P. M. (2002). The Obsessive-Compulsive Inventory: Development and validation of a short version. Psychological Assessment, 14, 485-496. doi:10.1037/1040-3590.14.4.485

Foa, E. B., Kozak, M. J., Salkovskis, P. M., Coles, M. E., \& Amir, N. (1998). The validation of new Obsessive-Compulsive Disorder Scale: the Obsessive-Compulsive Inventory. Psychological Assessment, 10, 206-214. doi:10.1037/1040-3590.10.3.206

Fullana, M. A., Tortella-Faliu, M., Caseras, X., Andion, O., Torrubia, R., \& Mataix-Cols, D. (2005). Psychometric properties of the Spanish version of the Obsessive-Compulsive Inventory-Revised in a non-clinical sample. Anxiety Disorders, 19, 893-903. doi:10.1016/j.janxdis.2004.10.004

Ghassemzadeh, H. Bolhari, J., Birashk, B., \& Salavati, M. (2005b). Responsibility attitude in a sample of Iranian obsessive- compulsive patients. International Journal of Social Psychiatry, 51, 13-22. doi: $10.1177 / 0020764005053266$

Ghassemzadeh, H., Khamseh, A., \& Ebrahimkhani, N. (2005a). Demographic variables and clinical features of obsessive-compulsive disorder in Iran: A second report. In B. E. Ling, (Ed.), Obsessive-Compulsive Disorder Research (pp. 243-271). New York: Nova Science Publishers, Inc.

Ghassemzadeh,H., Mojtabai,R., Karamghadiri, N., \& Ebrahimkhani, N. (2005c). Psychometric properties of a Persian-Language version of the Beck Depression Inventory-Second edition: BDI-II-Persian. Depression and Anxiety, 21,185-192. doi:10.1002/da.20070

Ghassemzadeh, H., Mojtabai, R., Khamseh, A., Ebrahimkhani, N., Isazadegan, A. A., \& Saif-Nobakht, Z. (2002). Symptoms of obsessive-compulsive disorder in a sample of Iranian patients. International Journal of Social Psychiatry, 48, 20-28. doi:10.1177/002076402128783055

Goodman, W. K., \& Price, L. H. (1998). Rating scales for obsessive-compulsive disorder. In M. A. Jenike, L.Bear, and W. E. Minichiello (Eds.), Obsessive-compulsive disorders-practical management (pp. 97-117). St. Louis: Mosby.

Hajcak, G., Huppert, J. D., Simons, R. F., \& Foa, E. B. (2004). Psychometric properties of the OCI-R in a college sample. Behavior Research \& Therapy, 42, 115-123. doi:10.1016/j.brat.2003.08.002

Hodgson, R. J., \& Rachman, S. J. (1977). Obsessional-compulsive complaints. Behavior Research and Therapy, 15, 389-395. doi:10.1016/0005-7967(77)90042-0

Hu, L. T., \& Bentler, P. M. (1999). Cutoff criteria for Fit Indexes in covariance structural analysis: Conventional criteria versus new alternatives. Structural Equation Modeling, 6, 1-55. doi:10.1080/10705519909540118

Rachman, S. J., \& Hodgson, R. J. (1980). Obsessions and compulsions. Englewood Cliffs, NJ: Prentic-Hall.

Smari, L., Olason, D. T., Eyprosdottir, A., \& Frolunde, M. (2007). Psychometric properties of the obsessive compulsive inventory-revised among Icelandic college students. Scandinavian Journal of Psychology, 48, 127-133. doi:10.1111/j.1467-9450.2007.00574.x

St. Clare, T. (2003). Assessment procedures. In R. G. Menzies and P. de Silva (Eds), Obsessive- compulsive disorder: Theory, research and treatment (pp. 239-257). West Sussex: John Wiley.

Taylor, S. (1995). Assessment of obsessions and compulsions: Reliability, validity, and sensitivity to treatment effects. Clinical Psychology Review, 15, 261- 296. doi:10.1016/0272-7358(95)00015-H 